\title{
Validation of the COSMO-RS method: Six Binary Systems
}

\author{
Frank Eckert* and Andreas Klamt \\ COSMOlogic GmbH \& Co. KG, Burscheider Str. 515, D-51381 Leverkusen, Germany \\ * Corresponding author, Tel.: +49-2171-731682, e-mail: frank.eckert@cosmologic.de
}

\begin{abstract}
COSMO-RS, a general, very fast and easy-to-use methodology for the a priori prediction of thermodynamic data of liquids is applied to six binary mixtures for which recent accurate experimental vapor-liquid- and liquid-liquid-equilibria data are available (Giles, N. F.; Wilson, G. M.; J. Chem. Eng. Data, 2000, 45, 146). The quality and applicability of COSMO-RS in the prediction of activity coefficients and vapor pressures is thus demonstrated. It is shown that COSMO-RS is an alternative to structure interpolating group contribution methods since it is independent of experimental data and generally applicable.
\end{abstract}

Keywords: COSMO, COSMO-RS, vapor-liquid equilibria, liquid-liquid equilibria, activity coefficient prediction, vapor pressure prediction. 


\section{Introduction}

The knowledge of the thermodynamic properties of solutions or mixtures of liquids is required in a large part of chemical engineering (for example in all kinds of separation processes, in dissolution of solids, crystallization from solution, absorption, adsorption, membrane processes, liquid-liquid-extraction processes, distillation, extractive or azeotropic distillation, solubility of gases in liquids or polymers, ... ). Theoretical and computational models can be valuable tools for the estimation of such properties, thus reducing time, resources and overall cost in process design. One can distinguish between two basic approaches to the computational prediction of thermodynamic data of mixtures. First, methods based upon inter- or extrapolation of a given set of experimental thermodynamic data for the regarded system, which are only applicable to systems where some thermodynamic data is already known (i.e. interpolating, "non- predictive" methods such as most activity coefficient models). Second, methods that are independent from experimental thermodynamic data of the given systems (i.e. "predictive"). The estimates are obtained from molecular structure information only. Predictive methods often are indispensable for chemical engineers in the synthesis and design of chemical processes and plants, since quite often no experimental data for certain compounds, neither from databases nor from measurement, are available at affordable costs for the mixtures regarded. Most prominent examples for the second approach are group contribution methods (GCMs) like UNIFAC ${ }^{1,2}$ or $\mathrm{ASOG}^{3}$ that are based on interaction parameters which have been obtained previously by analysis of phase equilibrium data of systems containing the same functional groups. Another method belonging to the second class is COSMO-RS" the "the conductor like screening model for realistic solvation" which is based solely upon unimolecular quantumchemical calculations of the individual species in the system (i.e. not of the mixture itself). The theory of COSMO-RS has been given elsewhere ${ }^{5,6}$ and will not be considered here. However we want to shortly sketch the practical course of a COSMO-RS calculation and stress the basic differences between COSMORS and GCMs: GCMs are based on the assumption that, with appropriately defined groups, the interaction energy of any system can be well approximated by the sum of functional group interaction energies. I.e. a liquid is considered not a mixture of interacting molecules but a mixture of interacting structural groups. The properties of a certain mixture can be predicted from previously determined group interaction parameters, which have been adjusted to available experimental data of systems containing the same functional groups. Thus GCMs critically depend upon the availability of the appropriate group interaction parameters and therefore the generality of their applicability is limited. The COSMO-RS approach starts from a very different 
point of view, namely from the complete molecule or - to be more precise - from the molecular surface as computed by quantumchemical methods (QM). COSMO-RS combines an electrostatic theory of locally interacting molecular surface descriptors (which are available from QM calculations) with an exact statistical thermodynamics methodology ${ }^{5,6}$. In practice, each molecule that is involved in a mixture has to be computed by the quantumchemical "Conductor-Like Screening Model" (COSMO) $)^{7,8}$. Such calculations are well done over night on single CPU in most cases but can be quite time consuming for very large molecules. However they have to be done only once per molecule and can be stored in a database and subsequently used from there. The subsequent COSMO-RS calculation that predicts the thermodynamic properties (e.g. chemical potentials, activity coefficients, solvent partition coefficients, solubilities, vapor pressures, excess Gibbs free energies, excess enthalpies, ...) is done in a few seconds, thus is also appropriate to the task of screening a large number of compounds from a database. COSMO-RS depends on the extremely small number of sixteen adjustable parameters some of which are physically predetermined ${ }^{6}$. COSMO-RS parameters are not specific regarding functional groups or molecule types. The parameters have to be optimized only for the QM-COSMO method that is to be used as a basis for the COSMO-RS calculations. Thus the resulting parametrization is completely general and can be used to predict the properties of almost any imaginable compound mixture or system (including even experimentally unavailable entities such as reactive intermediates or transition state structures). This is the main practical difference of the COSMO-RS method to GCMs. Currently the quality of the COSMO-RS predictions can not reach that of GCMs in their "core-region" in which they are well parametrized (i.e. VLE data of simple organic solvents). However, often COSMO-RS predictions are of similar quality or can be applied to problems where GCMs cannot be used at all (e.g. due to unknown interaction parameters, unknown group increments or proximity effects). This account attempts to demonstrate and validate the applicability and quality of the COSMO-RS method in predicting mixture phase equilibrium data of chemically diverse systems.

\section{Applications}

This section presents a variety of COSMO-RS applications to practical thermodynamic problems, namely the prediction of VLE and LLE data for systems recently measured by Giles and Wilson ${ }^{9}$ in connection with project 805 of the Design Institute for Physical Property Data (DIPPR) of the American Institute of Chemical Engineers. The systems measured are of high relevance to industry and represent typical applications for methods that a priori predict thermodynamic data 
such as COSMO-RS. Therefore, the measurements of Giles and Wilson have been chosen to validate the COSMO-RS method on a practically relevant set of experimental data.

All of the COSMO-RS calculations have been done using the COSMOtherm program ${ }^{10}$. The underlying quantumchemical calculations of the molecular COSMO surfaces have been done with the Turbomole program package using B-P density functional theory with TZVP quality basis set $^{8}$. In order to allow a comparison with a GCM method, the systems have also been predicted by UNIFAC. A UNIFAC program that is based on the original UNIFAC model11 has been used $^{12}$. Please note that due to missing group interaction parameters not all of the systems could be predicted by UNIFAC.

\subsection{Vapor-Liquid-Equilibria}

The results of the VLE predictions are described below. COSMO-RS predictions are compared with the experimental values of Giles and Wilson'. They are presented in pressure vs. liquid resp. gas phase mole fraction composition (pxy) diagrams in Figures 1-5. The total pressures $p_{t o t}$ presented in Figures 1-5 have been obtained from

$$
p_{t o t}=p_{1}^{0} x_{1} \gamma_{1}+p_{2}^{0} x_{2} \gamma_{2}
$$

where $p_{i}^{0}$ are the pure compound vapor pressures (experimental values of Giles and Wilson cf. Table 9 of Ref.') for compounds $i(i=1,2), x_{i}$ are the mole fractions of the compounds in the liquid and $\gamma_{\mathrm{i}}$ are the activity coefficients of the compounds as predicted by COSMO-RS or UNIFAC. In any case ideal behavior of the gas phase has been assumed. Gas phase pressures were not corrected by fugacity coefficients. The vapor mole fractions $y_{i}$ have been calculated from the ratio of partial and total vapor pressure:

$$
y_{i}=p_{i}^{0} x_{i} \gamma_{i} / p_{t o t}
$$

Please note that COSMO-RS can also do a "full" a priori prediction of VLE data since it is also capable of predicting the pure compound vapor pressures $p_{i}^{0}$. However, in this case an additional source of error would be introduced into eqs. (1) and (2). In order to make available a measure of the quality of the "full" a priori calculation of VLE by COSMO-RS the COSMO-RS predictions of the $p_{i}^{0}$ for all compounds and temperatures of the regarded VLE systems are given in Table 1. 
The typical maximum error of COSMO-RS in the prediction of pure compound vapor pressures can be expected to be in the range of $0.5 \log \left(p_{V}\right)$ units ${ }^{5,6}$. The errors of the predictions presented in Table 1 are all well below this value - with the exception of hydrogen chloride showing a deviation of $\sim 0.48 \log \left(\phi_{V}\right)$ units at both temperatures. The root mean squares deviation of all predicted values is $0.30 \log \left(p_{V}\right)$ units. It can be concluded that the quality of a "full" a priori prediction of VLE (including the prediction of pure compound vapor pressures $p_{i}^{9}$ ) with COSMO-RS is considerably lower than the prediction of activity coefficients only. Thus, if possible, experimental pure compound vapor pressures should be used in COSMO-RS predictions of VLE phase diagrams. COSMO-RS' "full" a priori prediction of VLE data can be valuable as first qualitative guess and/or if no experimental data on the pure compounds are available or for a qualitative consistency check of experimental data. Please note that COSMORS' pure compound vapor pressure prediction does not require any additional or different parameters than the activity coefficient prediction - it is also included in the generic COSMO-RS approach. GCMs like UNIFAC on the other hand do not offer any means of predicting pure compound vapor pressures and rely on experimental data or reasonable estimates of $p_{i}^{0}$. Thus to assure an unbiased comparison of the VLE predictions of COSMO-RS and UNIFAC the experimental pure compound vapor pressures $p_{i}^{0}$ of Giles and Wilson ${ }^{9}$ were used in eqs. (1) and (2) to predict the VLE systems presented in Figures 1-5.

Figure 1 shows the VLE data of the binary system phenol (1) - styrene (2) at temperatures $\mathrm{T}=333.15 \mathrm{~K}(\mathbf{A})$ and $\mathrm{T}=373.15 \mathrm{~K}(\mathbf{B})$. This system shows positive deviation from Raoult's law, which is also predicted by COSMO-RS. For both temperatures and over the whole range of mixture compositions qualitative and quantitative agreement between COSMO-RS and experiment is very good. At the lower temperature the COSMO-RS predictions are slightly worse than the UNIFAC predictions for the dew point curve but slightly better for the bubble point curve whereas for the higher temperature, the COSMO-RS predictions are slightly closer to the experiment for both bubble and dew point curves.

Figure 2 shows the VLE data of the binary system ethyl mercaptane (1) - n-butane (2) at temperatures $\mathrm{T}=323.15 \mathrm{~K}(\mathbf{A})$ and $\mathrm{T}=373.15 \mathrm{~K}(\mathbf{B})$. This system also shows positive deviation from Raoult's law. Again agreement between experimental values and COSMO-RS predictions are very good. For this system UNIFAC and COSMO-RS predictions were almost identical.

Figure 3 shows the VLE data of the binary system tert-butyl mercaptane (1) - n-propane (2) at temperatures $\mathrm{T}=283.15 \mathrm{~K}(\mathbf{A})$ and $\mathrm{T}=333.15 \mathrm{~K}(\mathbf{B})$. This system exhibits positive deviation from 
Raoult's law in the tert-butyl mercaptane rich region and a slight negative deviation in the $n$ propane rich region, which is more pronounced at the higher temperature. Although the deviations from Raoult's law are very small for this system COSMO-RS is able to predict this behavior correctly. Due to the lack of adequate groups for tert-butyl mercaptane no UNIFAC predictions could be done for this system.

Figure 4 shows the VLE data of the binary system dimethyl ether (1) - n-propane (2) at temperatures $\mathrm{T}=273.15 \mathrm{~K}(\mathbf{A})$ and $\mathrm{T}=323.15 \mathrm{~K}$ (B). For this system the experimental measurements indicate the presence of a minimum-boiling point azeotrope at both temperatures in the dimethyl ether rich region. Unfortunately the authors of the experimental work do not give detailed information about the location of the azeotrope ${ }^{9}$. An azeotropic point is also predicted by COSMO-RS, namely for the composition $x_{1}=0.11$ at $\mathrm{T}=273.15 \mathrm{~K}$ and for $x_{1}=0.18$ at $\mathrm{T}=323.15 \mathrm{~K}$. In addition, the positive deviation from Raoult's law found experimentally was also predicted by COSMO-RS to a good quantitative agreement. For this system the UNIFAC predictions differed significantly from the COSMO-RS results. At both temperatures UNIFAC overestimated the positive deviation from Raoult's law much stronger than COSMO-RS. The azeotrope was also predicted by UNIFAC, namely for compositions $x_{1}=0.15$ at $\mathrm{T}=273.15 \mathrm{~K}$ and $x_{1}=0.22$ at $\mathrm{T}=323.15 \mathrm{~K}$.

Figure 5 shows the VLE data of the binary system trifluoro acetic acid (1) - hydrogen chloride (2) at temperatures $\mathrm{T}=258.15 \mathrm{~K}(\mathbf{A})$ and $\mathrm{T}=278.15 \mathrm{~K}(\mathbf{B})$. In contrast to the previous systems, which involved only small to moderately polar compounds, the two compounds involved in this system are very polar. Further, they allow for strong intermolecular hydrogen bonding. Thus it represents a chemically completely different situation in the mixture. Nevertheless, also in this case COSMO-RS is able to predict the VLE data in good agreement with experiment. Due to the lack of appropriate groups for hydrogen chloride no UNIFAC predictions could be done for this system. 


\subsection{Liquid-Liquid-Equilibria}

The liquid-liquid-equilibrium properties have been calculated from the equity

$$
x_{i}^{I} \gamma_{i}^{I}=x_{i}^{I I} \gamma_{i}^{I I}
$$

where indices $I$ and $I I$ denote the liquid phases.

Table 2 presents the liquid-liquid-equilibrium data for the binary system dimethylcarbonate (1) water (2) at temperatures $\mathrm{T}=293.15 \mathrm{~K}$ and $\mathrm{T}=333.15 \mathrm{~K}$. At both temperatures this system exhibits a region of immiscibility. For both temperatures COSMO-RS is able to predict this miscibility gap. In addition, the quantitative correspondence between experiment and COSMORS regarding the mole fractions of the liquids demixing is excellent for both temperatures. The temperature trend of the region of immiscibility is also predicted correctly. Due to the lack of appropriate groups for dimethylcarbonate no UNIFAC predictions could be done for this system. It should be noted that GCMs often have problems in the prediction of LLE properties (only recently have they been enhanced towards and parametrized for the reasonable prediction of LLEs $^{2}$ ). In contrast, due to its generic approach COSMO-RS is able to predict LLE properties without any extra parametrization or adjustment and with a quality comparable to that of VLE predictions.

\section{Conclusions}

COSMO-RS is a novel approach for the a priori computational prediction of equilibrium thermodynamic properties of pure compounds and arbitrary mixtures as has been demonstrated in the previous sections at VLE properties such as activity coefficients and vapor pressures and LLE properties such as miscibility of liquids (as computed from compound activity coefficients in a mixture). The COSMO-RS method has been applied to six binary systems (five VLE and one LLE system) for which very accurate experimental measurements are available? Although no special parameters have been adjusted for these systems and although the systems are chemically very different (ranging from systems with only weakly polar compounds such as ethylmercaptane with $n$-butane to extremely polar systems that also show hydrogen bonding such as 
trifluoroacetic acid with hydrogen chloride) in all cases the correspondence between experiment and the COSMO-RS predictions is very good, qualitatively as well as quantitatively. The GCM UNIFAC due to lack of appropriate groups was not applicable to three of the six systems presented. For the remaining systems, the quality of the UNIFAC results was similar or (in one case) worse than COSMO-RS. In the predictions of VLE properties COSMO-RS' resp. UNIFAC's activity coefficients have been used in combination with experimental values of the pure compound vapor pressures. Full a priori predictions of VLE data (including COSMO-RS prediction of the pure compound vapor pressures) showed a somewhat lower quality of the predicted properties. The deviations of the predicted pure compound vapor pressures were below $0.5 \log \left(p_{V}\right)$ units with a root mean squares deviation of $0.30 \log \left(p_{V}\right)$ units. Nevertheless, such full a priori predictions can be useful as a first guess on VLE data or if no experimental vapor pressure data is available. Please also note that GCMs are generally not capable of doing pure compound vapor pressure predictions. It can be concluded that COSMO-RS is an alternative and/or supplement to group contribution methods, which currently are widely used for such calculations. It must be noted that COSMO-RS is not yet applicable to systems near or beyond the critical region. Thus the system methyl-benzyl alcohol - hydrogen at elevated pressures that was also measured by Giles and Wilson ${ }^{9}$ could not be computed by COSMO-RS. However currently an extension of the COSMO-RS approach towards the critical region is being implemented and will be available soon ${ }^{13}$. 


\section{Nomenclature}

COSMO conductor-like screening model

COSMO-RS conductor-like screening model for real solvents

GCM group contribution method

LLE liquid-liquid equilibrium

$P_{i}^{0} \quad$ vapor pressure of pure compound $i$

$p_{\text {tot }} \quad$ total pressure, $\mathrm{kPa}$

VLE vapor-liquid equilibrium

$x_{i} \quad$ mole fraction of component $i$ in the liquid phase

$y_{i} \quad$ mole fraction of component $i$ in the gas phase

$\gamma_{i} \quad$ activity coefficient of component $i$ 


\section{Literature Cited}

(1) Fredenslund, A.; Gmehling, J.; Rasmussen, P. Vapor Liquid Equilibria Using UNIFAC; Elsevier; Amsterdam, 1977.

(2) Gmehling, J.; Lohmann, J.; Jakob, A.; Li, J.; Joh, R. A Modified UNIFAC (Dortmund) Model. 3. Revision and Extension. Ind. Eng. Chem. Res. 1998, 37, 4876.

(3) Kojima, K.; Tochigi, T. Prediction of Vapor-Liquid Equilibrium by the ASOG Method; Elsevier; Amsterdam, 1979.

(4) Klamt, A. Conductor-like Screening Model for Real Solvents: A New Approach to the Quantitative Calculation of Solvation Phenomena. J. Phys. Chem. 1995, 99, 2224.

(5) Klamt, A.; Jonas, V.; Buerger, T.; Lohrenz, J. C. W. Refinement and Parametrization of COSMO-RS. J. Phys. Chem. A, 1998, 102, 5074.

(6) Klamt A.; Eckert, F. COSMO-RS: A Quantum Chemistry Based Alternative to Group Contribution Methods for the Prediction of Activity Coefficients in Multi-Component Mixtures. Fluid Phase Equilibria, 2000, 172, 43.

(7) Klamt, A.; Schüürmann, G. COSMO: A New Approach to Dielectric Screening in Solvents with Explicit Expressions for the Screening Energy and its Gradient. J. Chem. Soc. Perkin Trans.2, 1993, 799.

(8) Schäfer, A.; Klamt, A.; Sattel, D.; Lohrenz, J. C. W.; Eckert, F. COSMO Implementation in TURBOMOLE: Extension of an efficient quantum chemical code towards liquid systems. Phys. Chem. Chem. Phys., 2000, 2, 2187.

(9) Giles, N. F.; Wilson, G. M. Phase Equilibria on Seven Binary Mixtures. J. Chem. Eng. Data, 2000, $45,146$.

(10) Klamt, A.; Eckert, F. COSMOtherm, Version C1.1-Revision 05.00; COSMOlogic GmbH \& Co KG, Leverkusen, Germany, 2000; for further information see URL: http: / / www . cosmologic . de. (11) Tiegs, D.; Gmehling, J.; Rasmussen, P.; Fredenslund, A. Vapor-Liquid Equilibria by UNIFAC Group Contribution: Revision and Extension. 4. Ind. Eng. Chem. Res., 1987, 26, 159.

(12) A freely distributed program based on the fourth revision of the original UNIFAC model ${ }^{11}$ has been used to do the UNIFAC predictions. The program is described in detail in S. Sandlers book ${ }^{14} \mathrm{p}$. 470. The program code is downloadable at the URL:

http://che.udel.edu/thermo/basicprograms.htm

(13) Klamt, A.; Eckert, F., COSMOlogic GmbH \& Co KG, unpublished results.

(14) Sandler, S. I., Chemical and Engineering Thermodynamics, 3 ${ }^{\text {rd }}$ Edition; John Wiley \& Sons; New York, 1999. 


\section{Figure Captions}

\section{Figure 1:}

Vapor-liquid-equilibria data of the binary system phenol (1) - styrene (2) at temperatures $\mathrm{T}=333.15 \mathrm{~K}(\mathbf{A})$ and $\mathrm{T}=373.15 \mathrm{~K}$ (B). Filled triangles resp. squares: Mole fractions in liquid $(x)$ resp. in gas phase (y), experimental values of Giles and Wilson'. Solid lines: Calculated values from COSMO-RS. Dotted lines: UNIFAC ${ }^{12}$.

\section{Figure 2:}

Vapor-liquid-equilibria data of the binary system ethyl mercaptane (1) - n-butane (2) at temperatures $\mathrm{T}=323.15 \mathrm{~K}(\mathbf{A})$ and $\mathrm{T}=373.15 \mathrm{~K}(\mathbf{B})$. Filled triangles resp. squares: Mole fractions in liquid $(x)$ resp. in gas phase $(y)$, experimental values of Giles and Wilson'. Solid lines: Calculated values from COSMO-RS. Dotted lines: UNIFAC ${ }^{12}$.

\section{Figure 3:}

Vapor-liquid-equilibria data of the binary system tert-butyl mercaptane (1) - n-propane (2) at temperatures $\mathrm{T}=283.15 \mathrm{~K}(\mathbf{A})$ and $\mathrm{T}=333.15 \mathrm{~K}(\mathbf{B})$. Filled triangles resp. squares: Mole fractions in liquid $(x)$ resp. in gas phase $(y)$, experimental values of Giles and Wilson'. Solid lines: Calculated values from COSMO-RS.

\section{Figure 4:}

Vapor-liquid-equilibria data of the binary system dimethyl ether (1) - n-propane (2) at temperatures $\mathrm{T}=273.15 \mathrm{~K}(\mathbf{A})$ and $\mathrm{T}=323.15 \mathrm{~K}$ (B). Filled triangles resp. squares: Mole fractions in liquid $(x)$ resp. in gas phase $(y)$, experimental values of Giles and Wilson'. Solid lines: Calculated values from COSMO-RS. Dotted lines: UNIFAC ${ }^{12}$.

\section{Figure 5:}

Vapor-liquid-equilibria data of the binary system trifluoro acetic acid (1) - hydrogen chloride (2) at temperatures $\mathrm{T}=258.15 \mathrm{~K}(\mathbf{A})$ and $\mathrm{T}=278.15 \mathrm{~K}(\mathbf{B})$. Filled triangles resp. squares: Mole fractions in liquid $(x)$ resp. in gas phase $(y)$, experimental values of Giles and Wilson'. Solid lines: Calculated values from COSMO-RS. 


\section{Tables}

Table 1:

A priori COSMO-RS predictions for pure compound vapor pressures. Experimental values: Giles and Wilson'. Calculated values: COSMO-RS.

Compound

Temperature $[\mathrm{K}]$

Vapor Pressure [kPa]

Experiment Calculated

\begin{tabular}{|c|c|c|c|}
\hline \multirow[t]{2}{*}{$n$-butane } & 323.15 & 496.3 & 1196.4 \\
\hline & 373.15 & 1525.5 & 3783.6 \\
\hline \multirow[t]{2}{*}{ tert-butyl-mercaptane } & 283.15 & 12.32 & 12.67 \\
\hline & 333.15 & 88.27 & 113.90 \\
\hline \multirow[t]{2}{*}{ dimethyl ether } & 273.15 & 266.3 & 363.3 \\
\hline & 323.15 & 1140.6 & 1815.8 \\
\hline \multirow[t]{2}{*}{ ethyl mercaptan } & 323.15 & 167.6 & 127.6 \\
\hline & 373.15 & 639.9 & 636.5 \\
\hline \multirow[t]{2}{*}{ hydrogen chloride } & 258.15 & 1720.3 & 569.9 \\
\hline & 278.15 & 2960.2 & 982.6 \\
\hline \multirow[t]{2}{*}{ phenol } & 333.15 & 0.660 & 0.872 \\
\hline & 373.15 & 5.482 & 6.488 \\
\hline \multirow[t]{4}{*}{ propane } & 273.15 & 474.2 & 1042.4 \\
\hline & 283.15 & 635.6 & 1448.7 \\
\hline & 323.15 & 1708.2 & 4120.4 \\
\hline & 333.15 & 2115.4 & 5072.6 \\
\hline \multirow[t]{2}{*}{ styrene } & 333.15 & 5.137 & 5.990 \\
\hline & 373.15 & 25.490 & 27.166 \\
\hline \multirow[t]{2}{*}{ trifluoroacetic acid } & 258.15 & 1.400 & 0.527 \\
\hline & 278.15 & 5.078 & 2.161 \\
\hline
\end{tabular}




\section{Table 2:}

Liquid-liquid-equilibrium data for the binary system dimethylcarbonate (1) - water (2) at temperatures $\mathrm{T}=293.15 \mathrm{~K}$ and $\mathrm{T}=333.15 \mathrm{~K}$. Experimental values: Giles and Wilson'. Calculated values: COSMO-RS.

$\mathrm{T}=293.15 \mathrm{~K}$

Liquid 1

Experiment Calculated

$\begin{array}{lll}\mathrm{x}_{1} & \mathbf{0 , 8 7 2 6} \quad 0,8985\end{array}$

$\mathrm{T}=333.15 \mathrm{~K}$

Liquid 1

Experiment Calculated

$x_{1} \quad 0,7608$

0,8545
Liquid 2

Experiment Calculated

0,0274

0,0213
0,0315

Liquid 2

Experiment Calculated

0,0226 
Figures

Figure 1
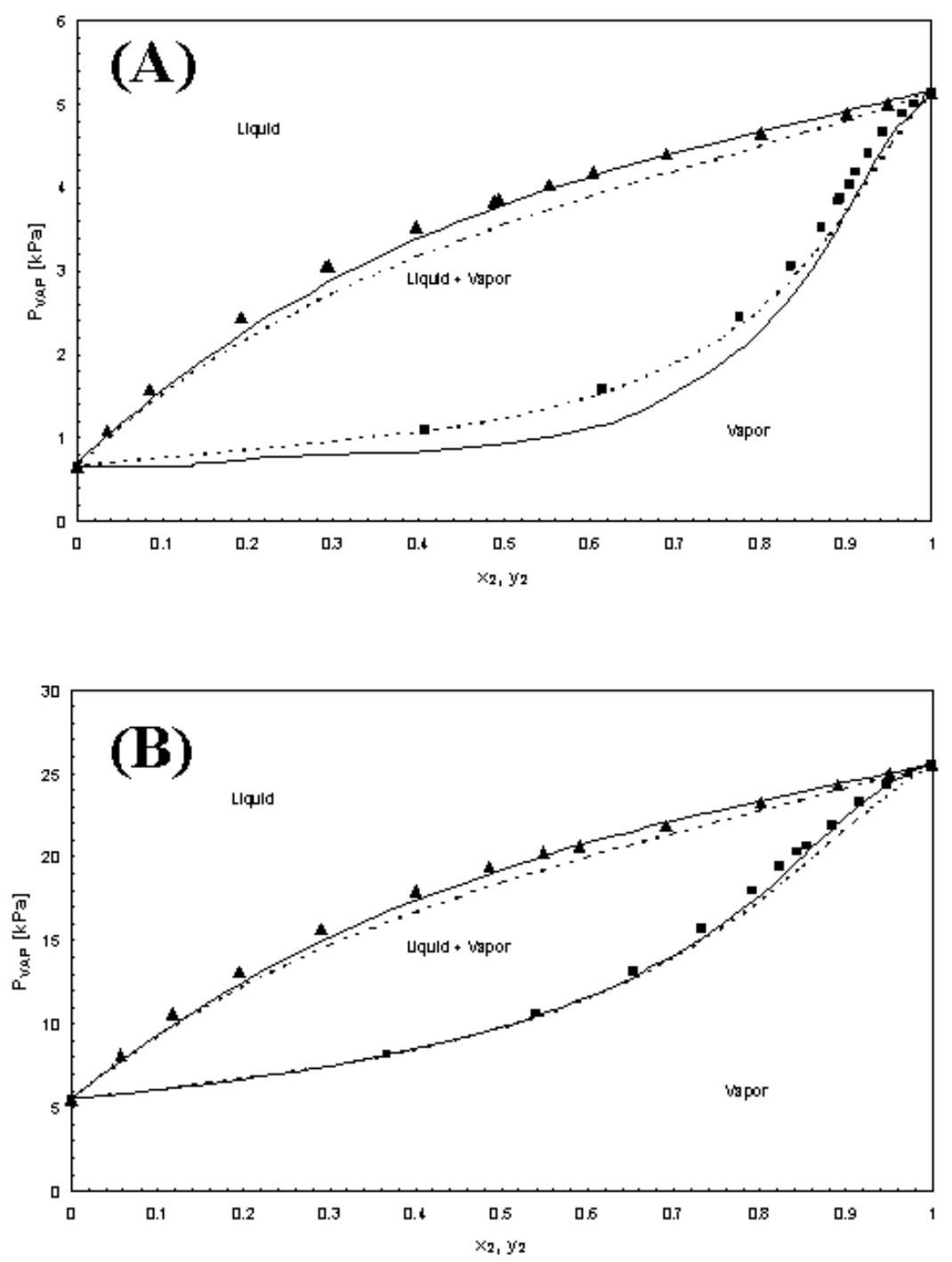
Figure 2
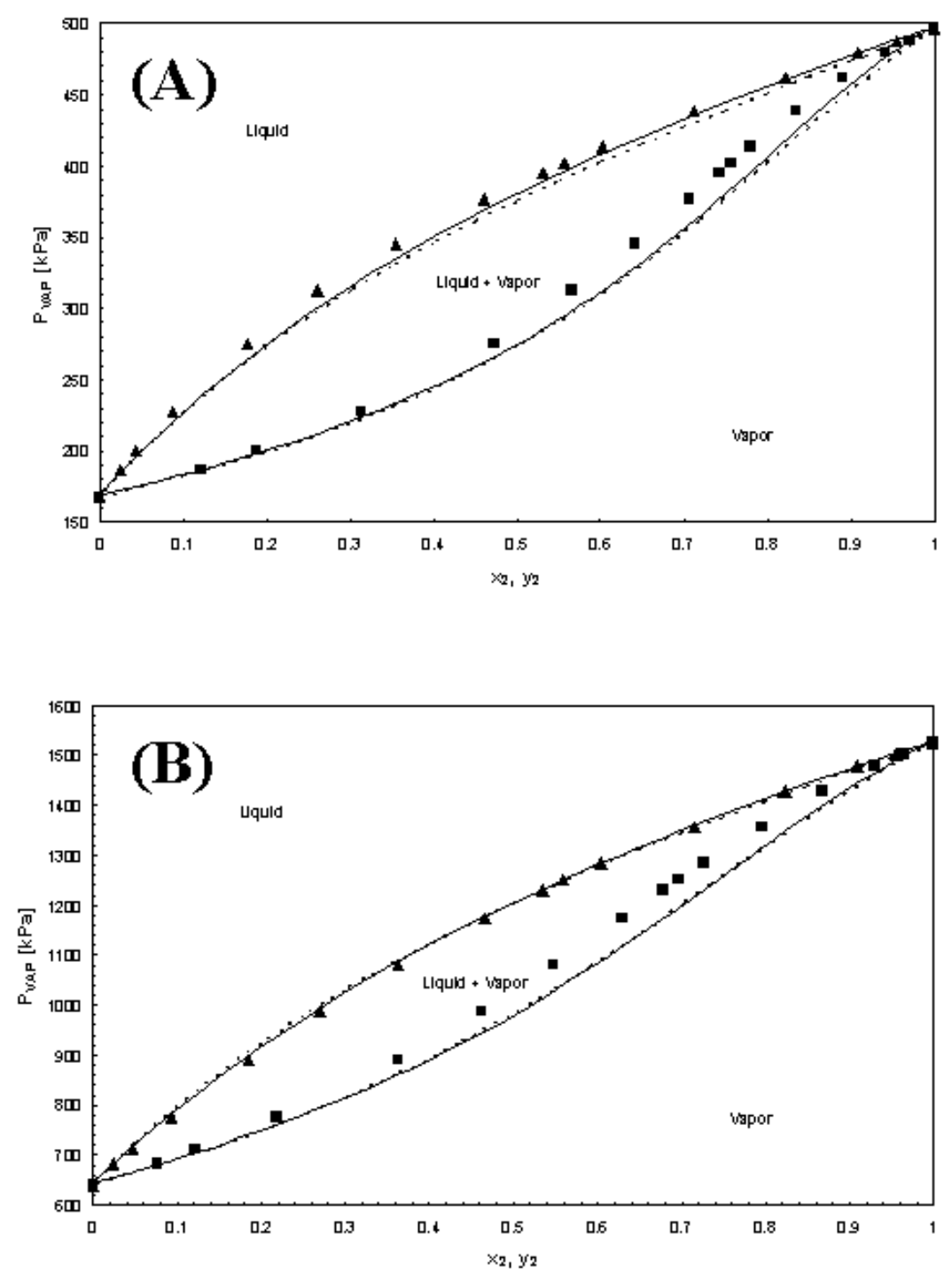
Figure 3
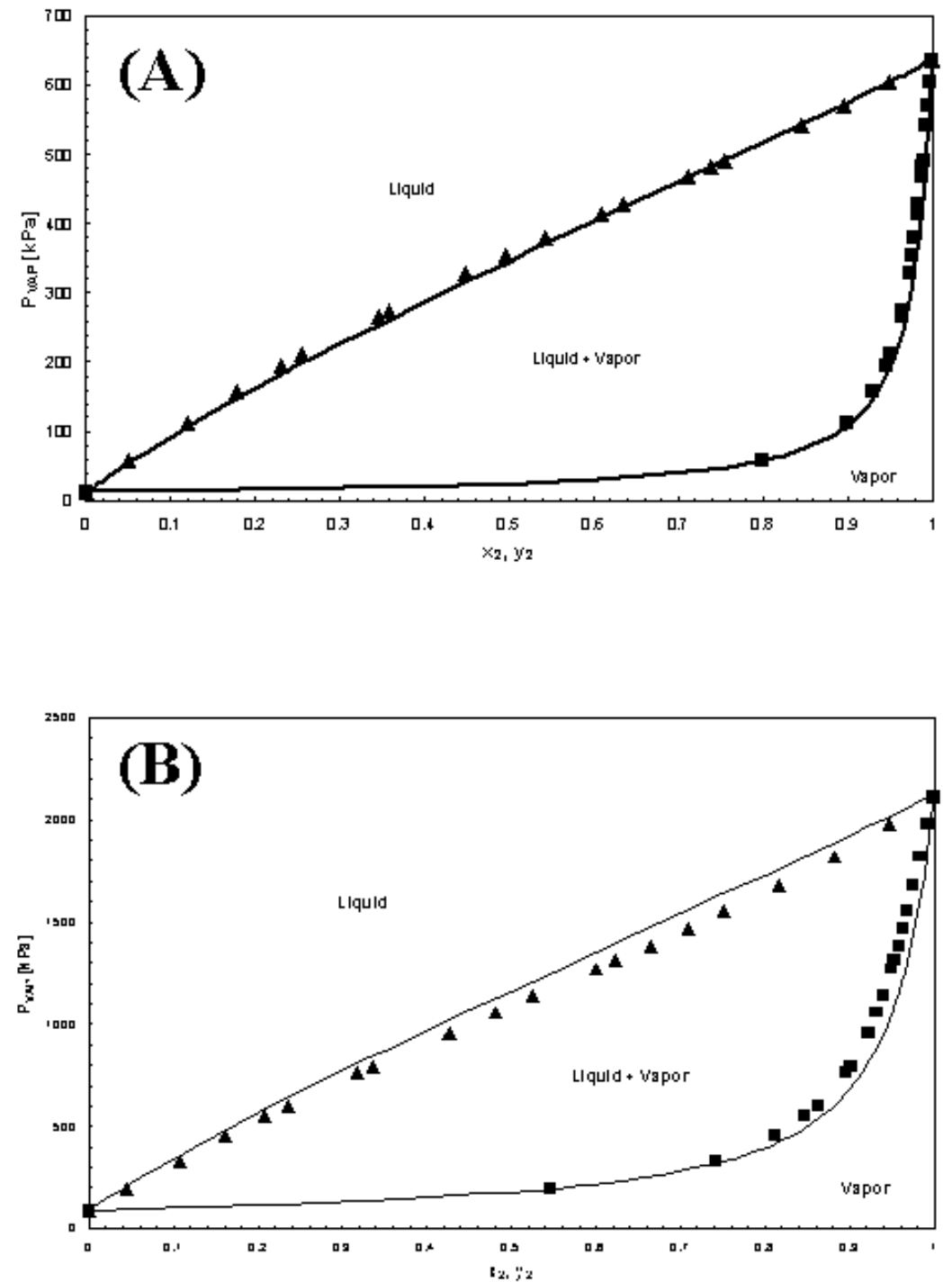
Figure 4
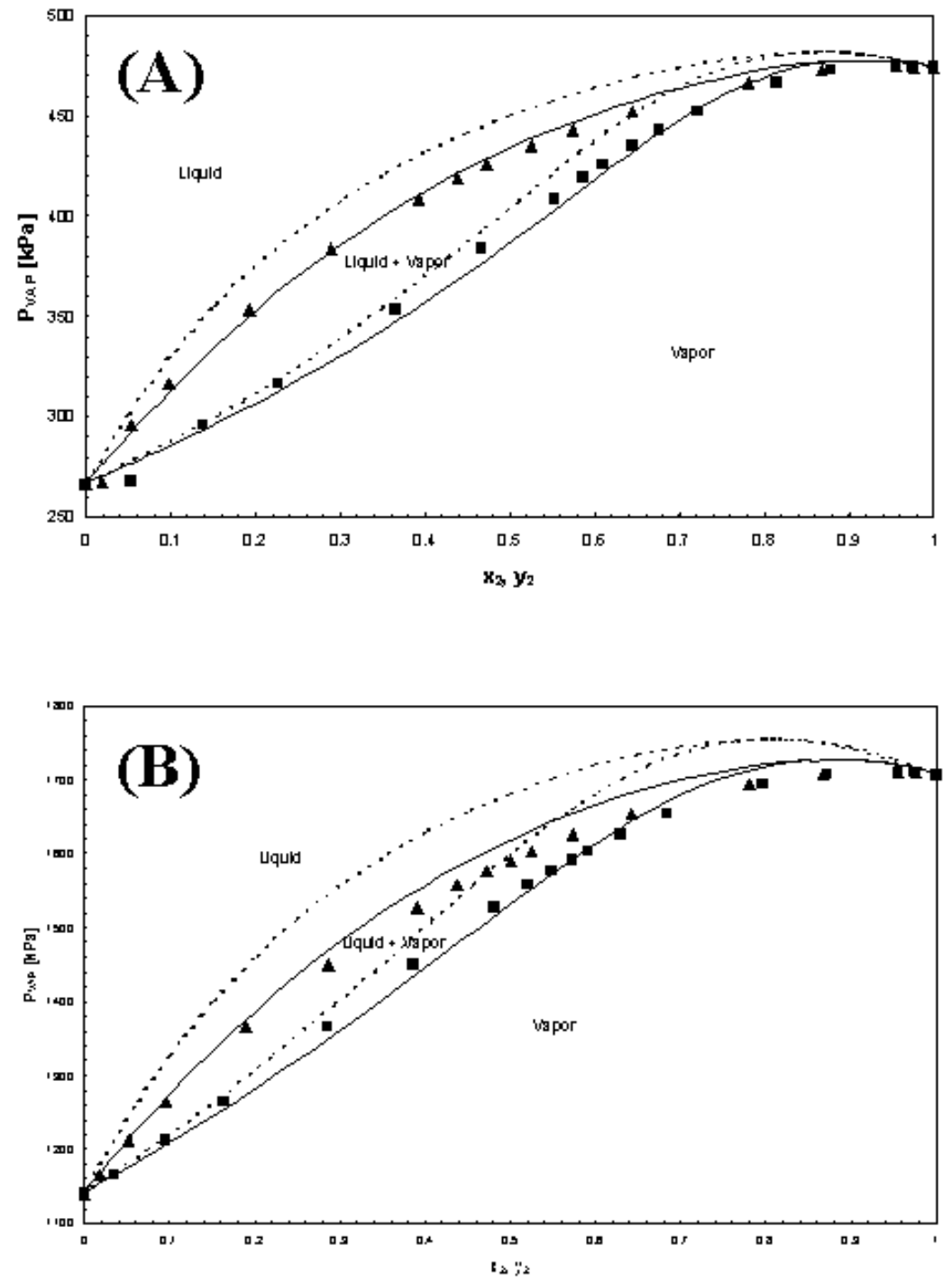
Figure 5
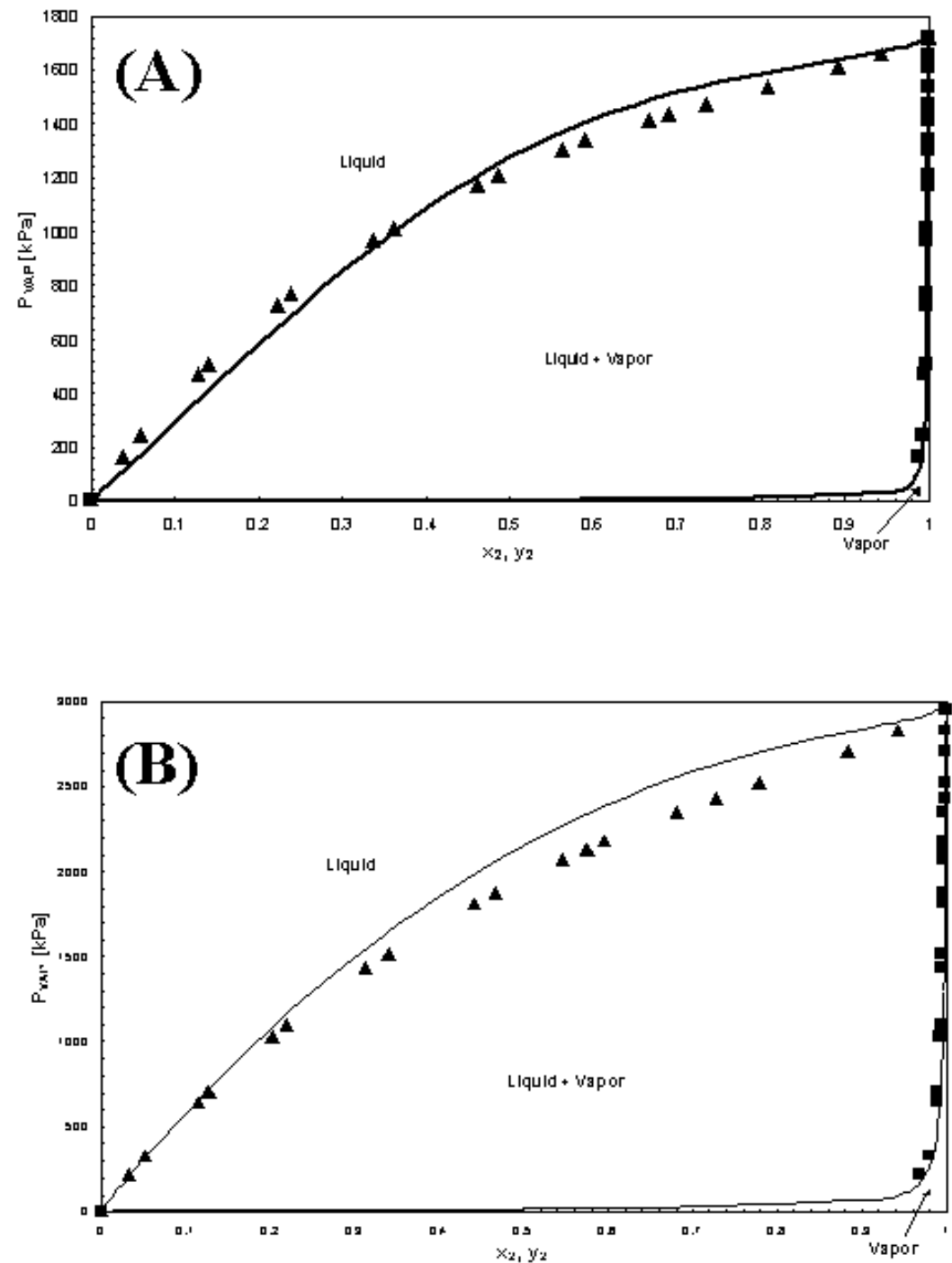
